Quim. Nova, Vol. 35, No. 5, 924-931, 2012

\title{
ESTUDIO DE LA CONTAMINACIÓN POR METALES EN SEDIMENTOS ACUÁTICOS DE LA BAHÍA DE MATANZAS
}

\author{
Odalys Quevedo, Jorge Gómez, Carmen Ramírez y Tamara Estrada \\ Centro de Investigaciones del Petróleo, CP 12000, La Habana, Cuba \\ Carlos Moreno y Carolina Mendiguchía \\ Departamento de Química Analítica, Facultad de Ciencias del Mar y Ambientales, Universidad de Cádiz, 11510 Puerto Real \\ (Cádiz), España \\ Margarita Edelia Villanueva Tagle* \\ Departamento de Química General, Facultad de Química, Universidad de la Habana, CP 10400, La Habana, Cuba \\ Mario Simeón Pomares Alfonso \\ Instituto de Ciencia y Tecnología de Materiales, Universidad de La Habana, CP 10400, La Habana, Cuba
}

Recebido em 28/8/11; aceito em 1/12/11; publicado na web em 28/2/12

\begin{abstract}
STUDY OF METAL CONTAMINATION IN AQUATIC SEDIMENTS OF THE BAY OF MATANZAS. Surface sediments of the Bay of Matanzas (Cuba) were studied to assess its environmental quality by using several criteria (metal content index, pollution load index and sediment quality guidelines). Two partial digestion sediment procedures and a modified BCR sequential extraction were used. The concentrations of metals were measured by atomic spectroscopy methods. The founded contents of $\mathrm{Cu}\left(2,4-27,9 \mathrm{mg} \mathrm{kg}^{-1}\right)$, $\mathrm{Zn}\left(2,5-55,5 \mathrm{~m} \mathrm{~kg}^{-1}\right)$ and $\mathrm{Ni}\left(8,8-99,2 \mathrm{mg} \mathrm{kg}^{-1}\right)$ were below those reported by other authors. The results obtained suggested that the most polluted sites were 3,5 , and 6 . The sequential extraction procedure showed that most of the studied metals were associated to the more stable fractions.
\end{abstract}

Keywords: metal pollution; marine sediments; digestion and extraction methods.

\section{INTRODUCCIÓN}

Cuba, debido a sus características geográficas particulares, posee amplias y diversas zonas costeras, cuya calidad ambiental, generalmente, se ve comprometida por los diferentes usos socio-económicos a que son destinadas. La Bahía de Matanzas es un ejemplo de ello. En investigaciones desarrolladas en esta zona se han evaluado alteraciones del contenido total en sedimentos de diversos metales pesados, tales como, $\mathrm{Cu}, \mathrm{Ni}$ y $\mathrm{Zn}$, entre otros, estableciéndose igualmente su posible origen. ${ }^{1-3}$ Sin embargo, no se conoce la fracción disponible de metales para los organismos vivos en el ecosistema. Además, no se ha evaluado la presencia de V, elemento de particular interés debido a la actividad que se desarrolla en esta bahía relacionada con la industria del petróleo cubana.

Es conocido que la contaminación por metales en sedimentos no puede ser evaluada sólo a partir de su contenido total, ya que la distribución de diversas formas químicas del metal en diferentes fases del sedimento, puede condicionar su mayor o menor movilidad, y por tanto la magnitud de su impacto sobre el ecosistema. ${ }^{4-6}$ Para evaluar de forma selectiva diferentes fracciones metálicas, diversos métodos de lixiviación parcial ácida de los metales, también llamados métodos de una sola etapa o métodos simples, han sido empleados en lugar de los métodos de extracción total. ${ }^{7-11}$ Varios de estos métodos constituyen normas internacionales (DIN 38414, EPA 3050 y 3051, ASTM D 3974 e ISO 11466, entre otros). En general, existe poca información sobre la relación entre las fases geoquímicas del sedimento disueltas y la eficiencia de extracción que se alcanza para cada elemento por un método específico. ${ }^{12,13} \mathrm{La}$ información que se obtiene sobre el contenido del metal varía en dependencia de la composición del sedimento, del elemento de interés y de la fortaleza del ataque que se realiza, ${ }^{12,14-16}$ por lo que resulta conveniente determinar la eficiencia

\footnotetext{
*e-mail: villa@fq.uh.cu
}

de extracción de los metales de interés en los sedimentos en estudio, junto al contenido total, para una mejor interpretación ambiental de los resultados. ${ }^{17}$

Otros métodos frecuentemente empleados en la evaluación de la contaminación por metales en sedimentos son los métodos secuenciales, conocidos también como métodos de varias etapas. ${ }^{6,18,19}$ La extracción química secuencial, aunque presenta imprecisiones, ${ }^{20}$ constituye una aproximación geoquímica y ambiental para comprender el comportamiento químico y la disponibilidad de los metales en el sedimento. ${ }^{6,21}$ Datos sobre la movilidad y biodisponibilidad potencial de los elementos obtenidos con estos procedimientos secuenciales resultan muy útiles en la estimación de riesgos ecológicos. ${ }^{22,23}$

Por otra parte, la determinación cuantitativa del grado de contaminación de un sedimento puede realizarse mediante el empleo de los llamados índices de contaminación. ${ }^{24}$ Existen diversos criterios basados en los coeficientes de enriquecimiento o factores de concentración, que permiten establecer el índice de contaminación en sedimentos. Entre estos criterios se encuentran el índice de carga contaminante (Pollution Load Index - PLI ${ }^{25}$ y el índice del contenido de metales (Metal Content Index - MCI). ${ }^{24-27}$ Las Guías de calidad de sedimentos también pueden ser empleadas para la evaluación del nivel de contaminación por metales. ${ }^{28}$ En ellas se establecen tres niveles de referencia para la concentración del metal: el nivel de efectos umbral (Threshold Effects Level - TEL), que es la concentración por debajo de la cual es de esperar que rara vez ocurran efectos adversos sobre la biota; el intervalo de bajos efectos (Effects Range Low - ERL) y el nivel de efectos probables (Probable Effects Level - PEL). Este último indica la concentración a partir de la cual es de esperar, con mayor probabilidad, la aparición de efectos adversos en la biota. Para algunos elementos citados en esta guía sólo aparecen reportados valores que provocan un efecto aparente.

El objetivo del presente trabajo es estudiar la contaminación por $\mathrm{Cu}$, $\mathrm{Ni}, \mathrm{V}$ y Zn en sedimentos superficiales de la Bahía de Matanzas a partir 
del conocimiento de su contenido total, de la eficiencia de extracción de estos metales por dos métodos simples de digestión parcial, el método ASTM y el método del ácido acético, y el estudio de la especiación de los metales en fracciones del sedimento con el método de extracción secuencial BCR (Bureau Community of Reference) modificado. Se emplearán diversos criterios recomendados para la evaluación de la contaminación. El $\mathrm{Cu}$ y Zn se estudiarán como indicadores de contaminación doméstica y urbana, mientras que el $\mathrm{Ni}$ y $\mathrm{V}$ como indicadores de contaminación industrial y de actividad petrolera en particular. ${ }^{29,30}$

\section{PARTE EXPERIMENTAL}

\section{Área de estudio}

La Bahía de Matanzas se ubica en la costa Norte de Cuba, a $23^{\circ}$ $03^{\prime}$ de latitud norte y $81^{\circ} 34^{\prime}$ de longitud oeste. Es una bahía amplia y abierta con un área aproximada de $36 \mathrm{~km}^{2}$ y un perímetro de $19 \mathrm{~km}$. Su profundidad media es de $200 \mathrm{~m}$. Los ríos Buey Vaca, Canimar, Yumurí y San Juan tributan a la Bahía. El último se destaca porque presenta el mayor escurrimiento y en su conjunto, constituyen fuentes de aporte de agua dulce y sedimentos con diferentes grados de contaminación. Otra fuente potencial de contaminación de la bahía la constituye el área portuaria-industrial que se ubica en su ribera noroeste, en la que se encuentran ubicadas diferentes industrias, destacándose entre las más importantes una tenería, una planta de fertilizantes, una base de almacenamiento de combustibles derivados del petróleo y una central termoeléctrica.

\section{Toma y preparación de muestras}

La red de estaciones consta de 9 sitios de muestreo (Figura 1S, material suplementario). Las estaciones 1 y 2 se ubican en la zona industrial, y la 3 y 4 en el área portuaria. Los sitios de muestreo del 5 al 8 se sitúan en una zona influenciada por vertimientos de aguas residuales, mientras que la estación 9, en la Playa Buey Vaca, constituye una estación de referencia alejada de la zona de influencia antropogénica.

Los sedimentos superficiales se colectaron con una draga Van Veen, de acuerdo a las normas ISO 5667/19, ${ }^{31}$ durante la estación de seca del año 2006. Para su traslado al laboratorio y preservación hasta el procesamiento, las muestras se colocaron en bolsas plásticas estériles y se congelaron inmediatamente después de colectadas. Así, se limitó la actividad biológica y se previno cualquier transformación química o alteraciones de la materia orgánica. ${ }^{32}$

Una vez en el laboratorio se descongelaron y sometieron al proceso de cuarteo, para lograr homogeneidad. Luego se secaron a $105^{\circ} \mathrm{C}$ hasta peso constante, se tamizaron con tamices plásticos, se seleccionó la fracción con tamaño de partículas inferior a $63 \mu \mathrm{m}$ y se mantuvo en recipientes plásticos estériles hasta su análisis.

\section{Reactivos y equipos}

Las disoluciones de referencia para la calibración de las técnicas de cuantificación empleadas se prepararon por dilución a partir de disoluciones de $1000 \mu \mathrm{g} / \mathrm{mL}$ (CertiPur, Alemania) de Cu, Ni, Zn y V. Los reactivos que se emplearon fueron de calidad analítica.

El contenido de los metales estudiados extraídos por los métodos de digestión simples fue cuantificado por espectrometría de absorción atómica con llama (FAAS, Flame Atomic Absorption Spectrometry), con un espectrómetro GBC Avanta $\Sigma$ (Australia). Las condiciones instrumentales utilizadas fueron las recomendadas por el fabricante (Tabla 1).

Las concentraciones en los extractos obtenidos con el método de extracción secuencial (BCR modificado) fueron determinadas mediante la espectrometría de masas con plasma inductivamente acoplado (ICP-MS, Inductively Coupled Plasma Mass Spectrometry) con el espectrómetro ICP-MS, X7 Series Thermo Elemental (Reino Unido). Las condiciones empleadas fueron: potencia de radio frecuencia aplicada de 1,4 kW, flujo del gas auxiliar de $0,80 \mathrm{~L} \mathrm{~min}^{-1}$, flujo del nebulizador de $0,93 \mathrm{~L} \mathrm{~min}^{-1}$, velocidad de introducción de la muestra de $0,4 \mathrm{~mL} \mathrm{~min}^{-1}$, etc. Los isótopos medidos fueron: $\mathrm{Cu}^{63}$, $\mathrm{Ni}^{60}, \mathrm{~V}^{51}$ y $\mathrm{Zn}^{66}$.

\section{Procedimientos de digestión}

Entre los diferentes métodos de digestión parcial reportados, dos serán considerados en este trabajo: el método $\mathrm{ASTM}^{10}$ y el método del ácido acético, ${ }^{33,34}$ ambos de amplio reconocimiento internacional. En el método ASTM, utilizado en los análisis ambientales de rutina de nuestros laboratorios, el sedimento es tratado con una mezcla de $\mathrm{HNO}_{3} \mathrm{y} \mathrm{HCl}$; mientras que el método del ácido acético, recomendado para estudios de biodisponibilidad, emplea una disolución al 25\% v/v de ácido acético. El método de digestión total propuesto por Ajlec, ${ }^{35}$ que usa una mezcla de $\mathrm{HNO}_{3}: \mathrm{HClO}_{4}: \mathrm{HF}$, será empleado en el presente trabajo para la determinación del contenido total de los elementos en el sedimento.

\section{Determinación del contenido total de metales}

A $0,3000 \pm 0,0001 \mathrm{~g}$ de sedimento seco se agregaron $2 \mathrm{~mL}$ de $\mathrm{HNO}_{3}(1: 1)$ en crisol de platino y se dejó en reposo durante $8 \mathrm{~h}$. Se adicionó $12 \mathrm{~mL}$ de una mezcla $1: 3$ de $\mathrm{HClO}_{4}$ y $\mathrm{HNO}_{3}$ y se evaporó en baño de arena hasta humos blancos. Se adicionaron $10 \mathrm{~mL}$ de $\mathrm{HF} 40 \%$ (Merck, Alemania) y se evaporó hasta sales húmedas. La adición de la mezcla $\mathrm{HClO}_{4}: \mathrm{HNO}_{3}$ y del $\mathrm{HF}$ se repitió dos veces. El residuo se disolvió con $3 \mathrm{~mL}$ de $\mathrm{HNO}_{3}(1: 1)$, se trasvasó cuantitativamente a un volumétrico de $25 \mathrm{~mL}$ y se enrasó con agua desionizada. La disolución se analizó por FAAS. La veracidad del procedimiento fue evaluada con los Materiales de Referencia Certificado (MRC) de sedimento marino: BCSS-1 y de sedimento estuarino IAEA-405, suministrados por el Nacional Research Council of Canadá y la International Atomic Energy Agency (IAEA), respectivamente.

\section{Determinación de la concentración de metales extraíbles por el} método ASTM

Se adicionó $50 \mathrm{~mL}$ de $\mathrm{H}_{2} \mathrm{O}$ destilada y $1 \mathrm{~mL}$ de $\mathrm{HNO}_{3} 69 \%$ (Merck, Alemania) a 0,5000 $\pm 0,0001 \mathrm{~g}$ de sedimento seco. Se añadió

Tabla 1. Parámetros instrumentales

\begin{tabular}{|c|c|c|c|c|c|c|}
\hline Elemento & $\begin{array}{l}\text { Longitud de onda } \\
(\mathrm{nm})\end{array}$ & $\begin{array}{l}\text { Flujo de acetileno } \\
\text { (L/min) }\end{array}$ & $\begin{array}{l}\text { Flujo de óxido nitroso } \\
(\mathrm{L} / \mathrm{min})\end{array}$ & $\begin{array}{l}\text { Ancho de banda } \\
(\mathrm{nm})\end{array}$ & $\begin{array}{l}\text { Altura del quemador } \\
\qquad(\mathrm{mm})\end{array}$ & $\begin{array}{c}\text { Corriente de lámpara } \\
(\mathrm{mA})\end{array}$ \\
\hline $\mathrm{Ni}$ & 232.0 & 1.40 & - & 0.2 & 17.4 & 4.0 \\
\hline $\mathrm{Cu}$ & 324.7 & 1.47 & - & 0.5 & 17.6 & 3.0 \\
\hline $\mathrm{V}$ & 318.3 & 6.00 & 10.0 & 0.2 & 18.2 & 10.0 \\
\hline $\mathrm{Zn}$ & 213.9 & 1.34 & - & 0.2 & 18.9 & 5.0 \\
\hline
\end{tabular}


$10 \mathrm{~mL} \mathrm{HCl} \mathrm{37 \%} \mathrm{(Merck,} \mathrm{Alemania)} \mathrm{y} \mathrm{se} \mathrm{calentó} \mathrm{a} 95{ }^{\circ} \mathrm{C}$ en placa de calentamiento hasta que el volumen se redujo a $10-15 \mathrm{~mL}$. Se enfrió, filtró y enrasó con agua desionizada en volumétricos de 25 $\mathrm{mL}$. Las concentraciones de los metales lixiviados se determinaron bajo las mismas condiciones descritas para la determinación de los contenidos totales.

Determinación de la concentración de metales extraíbles por el método de ácido acético

Se pesó en erlenmeyer 2,0000 $\pm 0,0001 \mathrm{~g}$ de sedimento seco, se adicionó $25 \mathrm{~mL}$ de ácido acético (HAc) al 25\% v/v (Merck, Alemania), se tapó y sometió a agitación moderada durante $6 \mathrm{~h}$ en una zaranda. Se transfirió cuantitativamente el contenido a tubos de centrífuga plásticos y se centrifugó a 2500 rpm durante 10 min. Se decantó el líquido sobrenadante en un volumétrico de $50 \mathrm{~mL}$, se lavó el residuo del tubo con $10 \mathrm{~mL}$ de $\mathrm{H}_{2} \mathrm{O}$ destilada y se centrifugó nuevamente. Se transfirió el sobrenadante al volumétrico y se enrasó con HAc al $25 \%$ v/v. La concentración se determinó bajo las mismas condiciones descritas para la determinación de las concentraciones totales, con la excepción de que las disoluciones de referencia para la calibración se prepararon en ácido acético al $25 \% \mathrm{v} / \mathrm{v}$.

Las digestiones fueron realizadas por triplicado para todos los métodos estudiados.

\section{Procedimiento de extracción secuencial BCR modificado ${ }^{19,36}$}

Para el fraccionamiento de los metales en el sedimento se aplicó el procedimiento BCR modificado que separa el contenido de metales en tres fracciones (excluyendo la fracción residual): fracción intercambiable (Fase1), reducible (Fase 2) y oxidable (Fase 3). A continuación se describe cada una de las etapas:

\section{Fase 1}

En un tubo de centrífuga de $100 \mathrm{~mL}$ se adicionaron 1,0000 $\pm 0,0001$ g de sedimento seco y $40 \mathrm{~mL}$ de disolución de ácido acético $0,11 \mathrm{~mol} / \mathrm{L}$, este se tapó y se agitó durante $16 \mathrm{~h}$ a $22 \pm 5^{\circ} \mathrm{C}$ a una velocidad de 30 $\pm 10 \mathrm{rpm}$. Se separó el extracto del residuo sólido por centrifugación a 3000 rpm durante 20 min y se decantó el líquido sobrenadante hacia un recipiente de polietileno. El recipiente se tapó y el extracto se guardó en refrigeración a una temperatura alrededor de $4{ }^{\circ} \mathrm{C}$ hasta su posterior análisis. El residuo fue lavado añadiendo $20 \mathrm{~mL}$ de agua bidestilada y agitando durante $15 \mathrm{~min}$, luego se centrifugó nuevamente a $3000 \mathrm{rpm}$ durante $20 \mathrm{~min}$. El sobrenadante se decantó y desechó, teniendo cuidado de no descartar ninguna porción del residuo sólido.

\section{Fase 2}

Al residuo de la fase 1 en el tubo de centrífuga se agregó $40 \mathrm{~mL}$ de disolución $0,1 \mathrm{~mol} / \mathrm{L}$ de cloruro de hidroxilamina recientemente preparada. El tubo se tapó y el residuo se re-suspendió mediante agitación manual. A continuación se siguió el mismo procedimiento de extracción descrito en la fase anterior.

\section{Fase 3}

Al residuo de la fase 2 en el tubo de centrífuga se adicionó, en pequeñas alícuotas para evitar pérdidas debidas a la reacción violenta, $10 \mathrm{~mL}$ de disolución de peróxido de hidrógeno 8,8 mol/L. El tubo se tapó ligeramente con su tapa y se digirió a temperatura ambiente durante $1 \mathrm{~h}$, con agitación manual ocasional. La digestión se continuó durante $1 \mathrm{~h}$ a $85 \pm 2{ }^{\circ} \mathrm{C}$ en un baño de agua. A continuación se redujo el volumen a menos de $3 \mathrm{~mL}$ por calentamiento adicional del tubo destapado y se adicionó una nueva alícuota de $10 \mathrm{~mL}$ de peróxido de hidrógeno $8,8 \mathrm{~mol} / \mathrm{L}$. Se calentó nuevamente el recipiente tapado a 85 $\pm 2^{\circ} \mathrm{C}$ y se digirió durante $1 \mathrm{~h}$. Se quitó la tapa y se redujo el volumen de líquido hasta cerca de $1 \mathrm{~mL}$. Se adicionó al residuo frío $50 \mathrm{~mL}$ de disolución de acetato de amonio 1,0 mol/L. Se tapó el tubo y se re-suspendió el residuo mediante agitación manual. A continuación se siguió el mismo procedimiento descrito en la fase 1.

La extracción secuencial se realizó por triplicado. La concentración de metales en la fase residual (fracción del sedimento no disuelta en las tres etapas descritas) se estimó por la diferencia entre el contenido total determinado experimentalmente en este trabajo y la suma de las concentraciones determinadas en las 3 etapas del BCR. La veracidad del procedimiento de extracción secuencial fue comprobada mediante el análisis del material de referencia certificado de sedimento de lago BCR-701, suministrado por el Institute for Reference Materials and Measurements.

\section{Procesamiento de los datos experimentales}

El PLI se determinó como la raíz n-sima del producto de los "n" $\mathrm{FC}$ (factores de concentración) considerados: PLI $=\left(\mathrm{FC}_{1} \times \mathrm{FC}_{2} \mathrm{x}\right.$ $\left.\mathrm{FC}_{3} \times \ldots \times \mathrm{FC}_{\mathrm{n}}\right)^{1 / \mathrm{n}}$, donde $\mathrm{n}$ es el número de metales considerados y los FC se definen como la concentración de un metal en un sedimento contaminado dividido por el valor normal en un ambiente no contaminado.

El MCI se expresó como: $\mathrm{MCI}=\left(\mathrm{C}_{1} \times \mathrm{C}_{2} \times \ldots \times \mathrm{C}_{\mathrm{n}}\right)^{1 / \mathrm{n}}$, donde $\mathrm{C}_{\mathrm{n}}$ equivale a la concentración de cada metal $(\mathrm{mg} / \mathrm{kg})$ y n es el número de metales analizados.

Para el análisis comparativo de las concentraciones totales obtenidas para los MRC con los valores certificados se empleó el "Test de Rangos Múltiples”. Para el procesamiento de los datos se empleó el paquete de programa Statgraphic Plus 5.1, empleando un valor de $\mathrm{p}<0,05$ para determinar la significancia estadística de los resultados.

\section{RESULTADOS Y DISCUSION}

\section{Evaluación de la contaminación según la concentración total de metales}

Como se observa (Tabla 2), no existen diferencias significativas para un nivel de $95 \%$ de confianza entre la concentración certificada y la experimental, lo que fundamenta la aplicación del procedimiento de digestión total seleccionado junto a la cuantificación por FAAS para la determinación del contenido total de los elementos.

Tabla 2. Concentración de metales en los materiales de referencia analizados (materia seca)

\begin{tabular}{cccccc}
\hline \multirow{2}{*}{ Muestra } & \multicolumn{4}{c}{ Concentración media \pm intervalo de confianza $\left(\mathrm{mg} \mathrm{kg}^{-1}\right)$} \\
\cline { 3 - 6 } & & $\mathrm{V}$ & $\mathrm{Ni}$ & $\mathrm{Zn}$ & $\mathrm{Cu}$ \\
\hline \multirow{2}{*}{ BCSS-1 } & $\mathrm{C}_{\text {exp }}{ }^{1}$ & $93,6 \pm 5,2$ & $55,3 \pm 3,6$ & $119 \pm 12$ & $18,5 \pm 2,7$ \\
& $\mathrm{C}_{\text {cer }}{ }^{2}$ & $93,4 \pm 4,9$ & $47,8 \pm 5,6$ & $114 \pm 6$ & $21,2 \pm 1,0$ \\
\multirow{3}{*}{ IAEA 405 } & $\mathrm{C}_{\text {exp }}{ }^{1}$ & $95,0 \pm 4,7$ & $33,5 \pm 3,2$ & $281 \pm 2$ & $51,2 \pm 3,1$ \\
& $\mathrm{C}_{\text {cer }}{ }^{2}$ & $96,9 \pm 2,1$ & $32,5 \pm 1,3$ & $279 \pm 8$ & $47,7 \pm 1,4$ \\
\hline
\end{tabular}

${ }^{1}$ Concentración experimental. ${ }^{2}$ Concentración certificada.

En la Tabla 3 se muestra la concentración total promedio junto a la desviación estándar de cada metal en las 9 estaciones estudiadas. Según las Guías de Evaluación para la Calidad de los Sedimentos $(\mathrm{SQAG})^{37}$ la concentración de Zn para todas las estaciones es inferior al TEL. Para el $\mathrm{Cu}$ se aprecian concentraciones similares o ligeramente superiores al TEL (18,7 $\left.\mathrm{mg} \mathrm{kg}^{-1}\right)$ sólo en las estaciones 3 $(27,9 \pm 1), 5(18,1 \pm 0,3)$ y $6(21,6 \pm 2,0)$, para el resto son inferiores. En el caso del V, la SQAG sólo reporta el valor de efecto aparente $\left(57,0 \mathrm{mg} \mathrm{kg}^{-1}\right)$, detectándose concentraciones superiores a este en las 
Tabla 3. Concentración total de metales en sedimentos de la Bahía de Matanzas y criterios para la evaluación de la contaminación

\begin{tabular}{|c|c|c|c|c|c|c|}
\hline \multirow{2}{*}{ Muestra } & \multirow{2}{*}{ MCI } & \multirow{2}{*}{ PLI } & \multicolumn{4}{|c|}{ Concentración media \pm desviación estándar $\left(\mathrm{mg} \mathrm{kg}^{-1}\right)$} \\
\hline & & & $\mathrm{V}$ & $\mathrm{Ni}$ & $\mathrm{Zn}$ & $\mathrm{Cu}$ \\
\hline 1 & 23,7 & 6,2 & $63,3 \pm 1,0$ & $11,2 \pm 0,5$ & $30,4 \pm 0,7$ & $14,7 \pm 0,1$ \\
\hline 2 & 26,2 & 6,8 & $65,0 \pm 2,1$ & $23,8 \pm 2,0$ & $25,5 \pm 3,0$ & $11,9 \pm 0,4$ \\
\hline 3 & 39,2 & 10,2 & $67,7 \pm 2,1$ & $22,6 \pm 1,0$ & $55,5 \pm 0,9$ & $27,9 \pm 1,0$ \\
\hline 4 & 30,2 & 7,9 & $45,5 \pm 2,1$ & $23,8 \pm 0,2$ & $46,5 \pm 0,9$ & $16,6 \pm 0,6$ \\
\hline 5 & 48,3 & 12,5 & $84,1 \pm 1.1$ & $98,3 \pm 0,3$ & $36,4 \pm 1,0$ & $18,1 \pm 0,3$ \\
\hline 6 & 54,1 & 13,51 & $85,4 \pm 1,1$ & $99,2 \pm 0,9$ & $40,4 \pm 1,0$ & $21,6 \pm 2,0$ \\
\hline 7 & 20,4 & 5,3 & $42,5 \pm 5,1$ & $16,6 \pm 0,5$ & $20,0 \pm 1,4$ & $12,4 \pm 0,5$ \\
\hline 8 & 8,3 & 2,2 & $<4,2$ & $9,1 \pm 0,3$ & $12,1 \pm 1,1$ & $10,4 \pm 0,3$ \\
\hline 9 & 3,9 & - & $<4,2$ & $8,8 \pm 0,4$ & $2,5 \pm 0,5$ & $2,4 \pm 0,2$ \\
\hline TEL & & & $57,0^{*}$ & 15,9 & 124 & 18,7 \\
\hline PEL & & & & 42,8 & 271 & 108 \\
\hline
\end{tabular}

*Nivel de Efecto Aparente. MCI: Metal Content Index, PLI: Pollution Load Index, TEL: Threshold Effects Level, PEL: Probable Effects Level.

estaciones 1-3 de la zona portuario industrial (63,3-67,7 $\left.\mathrm{mg} \mathrm{kg}^{-1}\right)$, así como en la 5 y $6\left(84,1-85,4 \mathrm{mg} \mathrm{kg}^{-1}\right)$. Estos altos niveles de $\mathrm{V}$ en las estaciones 1-3, 5 y 6 tributan al incremento de la contaminación por metales pesados en esos puntos.

La mayor variabilidad de la concentración se obtiene para el $\mathrm{Ni}$ que, en las estaciones 5 y 6, sobrepasa en más del doble el PEL. La acumulación de Ni en las citadas estaciones está asociada, fundamentalmente, con las descargas de desechos urbano e industriales no tratados completamente que aportan grandes volúmenes de lodos enriquecidos y que son acarreados principalmente, por los ríos Yumurí y San Juan. En el caso de la estación 3, la morfología de la Bahía en esa zona contribuye a la retención de contaminantes.

Los sedimentos de las estaciones 6, 5 y 3 son limo-arenosos (entre 11 y $22 \%$ de estos sedimentos tienen tamaño de partículas inferior a $0,1 \mathrm{~mm}$ ) por tanto poseen mayor superficie de contacto lo que favorece la contaminación.

El índice del contenido de metales oscila entre 3,9 y 54,1 (Tabla 3). De acuerdo a este indicador, se establece el siguiente orden decreciente de contaminación de las estaciones estudiadas: $6>5>3>4>2>1>7>8>9$. La estación 6 presenta mayor grado de contaminación, seguida por la 5 y la 3 , respectivamente.

Las estaciones 2, 1 y 7 pertenecen a un segundo grupo menos contaminado, de acuerdo a sus valores de MCI (20,4-26,2). Finalmente existe un tercer grupo de menor nivel de contaminación, integrado por las estaciones 8 y 9 , siendo esta última la menos contaminada de todas $(\mathrm{MCI}=3,9)$.

El índice de carga contaminante se calculó tomando como nivel de fondo el correspondiente a la estación de referencia 9. Los valores de PLI (Tabla 3) son superiores a 1 para todos los sedimentos, destacándose también por este índice los sitios de muestreo 6, 5 y 3 por presentar las mayores cargas contaminantes.

Los tres criterios empleados para evaluar los niveles de contaminación coinciden en que los sedimentos correspondientes a los sitios de muestreo 6,5 y 3 son los que se encuentran más contaminados, decreciendo el nivel de contaminación en ese mismo orden para estos sitios.

Aunque el vertimiento de residuales urbano-industriales tratados parcialmente constituye una de las principales fuentes de contaminación a la bahía, es necesario destacar que en la zona se desarrollan actividades de la industria petrolera que deben tenerse en cuenta.

La comparación de los resultados obtenidos para la concentración total de metales en los sedimentos en la bahía en estudio, indican que los contenidos de $\mathrm{Cu}\left(2,4-27,9 \mathrm{mg} \mathrm{kg}^{-1}\right), \mathrm{Zn}(2,5-55,5$ $\left.\mathrm{mg} \mathrm{kg}^{-1}\right)$ y $\mathrm{Ni}\left(8,8-99,2 \mathrm{mg} \mathrm{kg}^{-1}\right)$ son ligeramente inferiores a los reportados previamente por González, ${ }^{1}$ Tur ${ }^{2}$ y Ruíz ${ }^{3}$ que oscilaban en los intervalos de 17,6-296, 22,3-140 y 33-195 $\mathrm{mg} \mathrm{kg}^{-1}$ para $\mathrm{Cu}$, $\mathrm{Zn}$ y Ni, respectivamente. Por tanto es posible concluir que el nivel de contaminación de este ecosistema, respecto al contenido total de metales, ha disminuido discretamente en el tiempo en el período comprendido entre los años 1989 y 2006, a lo que ha contribuido entre otros factores, la textura de los sedimentos presentes, que en su mayoría clasifican como arenosos o limo-arenosos, y otros factores oceanográficos como corrientes, mareas y oleaje. ${ }^{2}$

\section{Métodos de digestión parcial de una etapa}

La reproducibilidad de las mediciones, expresada como la desviación estándar relativa de 3 determinaciones fue inferior al 5\% con el método ASTM y no superó el $6 \%$ con el método del ácido acético para todos los elementos. Debido a la ausencia en el mercado de materiales certificados de referencia para la fracción del elemento que extraen estos dos métodos, no fue posible su utilización en este trabajo.

Para estimar la eficiencia de extracción (EE) de los métodos de digestión parcial se calculó el porcentaje que representa el contenido del elemento extraído por cada método de extracción con respecto a su contenido total en el sedimento (Figura 1a-d).

La EE del método ASTM para el Zn oscila entre 40-100\% según la muestra (Figura 1a). Para los sedimentos de las estaciones 1, 3, 4 y 5 la extracción es mayor al 91\%; mientras que el método del ácido acético extrae generalmente menos, entre 22 y $70 \%$. La eficiencia de extracción es similar por ambos métodos para las estaciones 2, 6, 8 y 9; pero varía entre 40-72\% según la muestra.

Para el Ni (Figura 1b) el método ASTM extrae entre 40-90\% según la muestra, de modo que se alcanzan extracciones cercanas al 90\% para los sedimentos de las estaciones 1, 3, 5 y 7. La extracción de Ni por el método del ácido acético es menor que la del método ASTM y fluctúa entre 2 y $44 \%$ en función de la muestra. La mayor lixiviación (44\%) para el acético se obtuvo en la estación 8.

La eficiencia de extracción del método ASTM para el V (Figura 1c) es baja en general, inferior al $43 \%$. Sólo se extrae todo el V presente en la estación 8 , donde su concentración es relativamente poca $\left(<4,2 \mathrm{mg} \mathrm{kg}^{-1}\right)$ comparada con el resto de las estaciones $(42,5-85,4$ $\left.\mathrm{mg} \mathrm{kg}^{-1}\right)$. El método de ácido acético no extrae el $\mathrm{V}$ de ninguna de las muestras estudiadas.

La EE del método ASTM para el Cu (Figura 1d), oscila entre 27-76\%. La mayor extracción se obtuvo sólo para las estaciones 3 y 5 . Al igual que para el $\mathrm{Ni}$, los porcentajes de $\mathrm{Cu}$ extraídos por el 

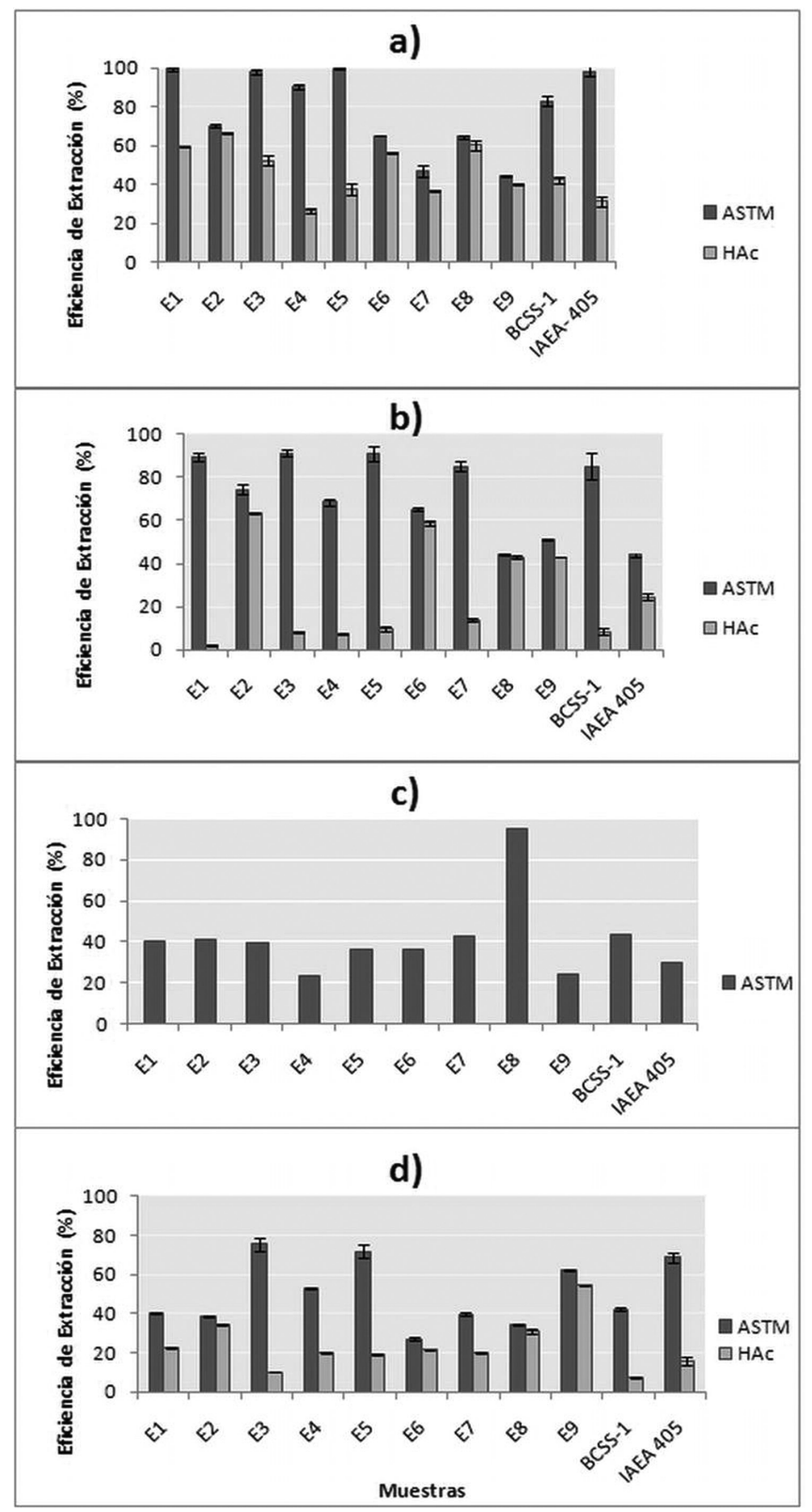

Figura 1. Eficiencia de extracción (\%) de los métodos ASTM y ácido acético, con respecto a la concentración total, de $\mathrm{Zn}(a), \mathrm{Ni}(b), V(c)$ y $\mathrm{Cu}(\mathrm{d})$ en los sedimentos de la Bahía de Matanzas y en los materiales de referencia BCSS-1 y IAEA-405

método del ácido acético son menores que por el método ASTM y fluctúan entre 10-26\%. Sólo en el caso de la estación 9 se logra una EE ligeramente superior al $40 \%$.

Estos resultados sugieren que, aunque la concentración de $\mathrm{V}$ en la mayoría de las estaciones supera la concentración de este elemento que puede producir efectos aparentes sobre la biota, según la SQAG, es poco probable que realmente represente una amenaza para el ecosistema en estudio. El V debe encontrarse en una forma química de elevada estabilidad o asociado a una fase del sedimento que impide su fácil extracción y por tanto debe ser difícil su introducción en la cadena trófica bajo condiciones ambientales, donde los procesos de lixiviación no deben ser más agresivos que los ensayados en este trabajo.

En general, teniendo en cuenta todos los sedimentos evaluados, la eficiencia de extracción del método ASTM decrece según el elemento en el orden siguiente: $\mathrm{Zn}(40-100 \%), \mathrm{Ni}(40-90 \%), \mathrm{Cu}(27-76 \%)$ y V $(<43 \%)$. Un comportamiento aproximadamente similar se observó para el método del ácido acético: Zn (22-70\%), Ni (2-44\%), Cu (10-26\%) y V (0\%).

También, es posible diferenciar en dos grupos las estaciones de muestreo según la eficiencia de extracción de los metales por los métodos de digestión parcial de una etapa estudiados. El método ASTM extrae aproximadamente el contenido total de $\mathrm{Zn}$ y Ni en los sedimentos de las estaciones 1, 3, 4 y 5, mientras que el método de ácido acético extrae menor cantidad. La EE del Cu en estas estaciones es inferior al $80 \%$. Por otra parte la cantidad de Zn, Ni y Cu extraída por ambos métodos en los sedimentos de las estaciones 2,6 y 8 es aproximadamente la misma, pero menor que el contenido total de esos elementos.

En los Materiales de Referencia Certificados (Figura 1) el método ASTM extrae aproximadamente el contenido total de $\mathrm{Zn}$ y Ni en el sedimento marino BCSS-1 y de $\mathrm{Zn}$ en el sedimento estuarino IAEA405, sin embargo la extracción Cu es inferior al 80\%. Las EE obtenidas por el método ASTM para $\mathrm{Zn}$, Ni y $\mathrm{Cu}$ en los MRC marcaron una diferencia significativa entre ambos materiales. $\mathrm{Al}$ igual que para las muestras en estudio, la extracción de $\mathrm{Zn}, \mathrm{Ni}$ y $\mathrm{Cu}$ por el método del ácido acético fue menor que por el método ASTM, dependiendo tanto del analito como de la muestra. La eficiencia de extracción del método ASTM también fue baja para el V, entre 30 y $45 \%$ según el material de referencia; mientras que el método de ácido acético no extrae $\mathrm{V}$ de los materiales de referencia, al igual que sucedió para las muestras de interés. En general, los dos MRC se comportan de forma diferente a los sedimentos de la bahía de Matanzas, ante el ataque ácido de los métodos de digestión simples empleados. Esto confirma la necesidad de conocer la eficiencia de extracción del método en los sedimentos colectados en el ecosistema en estudio. La extrapolación hacia los sedimentos de interés de resultados obtenidos en MRC, puede conducir a conclusiones erróneas. Como es conocido, el uso de materiales con el contenido total certificado de los metales resulta adecuado para evaluar la exactitud (reproducibilidad y veracidad) de la determinación total del elemento, pero no son útiles para los métodos de digestión parcial.

\section{Método BCR de extracción secuencial}

El método BCR de extracción secuencial fue aplicado a los sedimentos colectados en las estaciones 3,5 y 6 , donde los diferentes criterios empleados para evaluar la calidad de los sedimentos señalaron la mayor contaminación en el área de estudio, evaluada a partir del contenido total de elementos en el sedimento. Además, los sedimentos de las estaciones 3 y 5 por una parte y la estación 6 por otra, son representativos de los dos grupos establecidos de acuerdo a la eficiencia de extracción de los metales por los métodos de digestión parcial de una etapa previamente estudiados.

En la Tabla 4 se reportan las concentraciones de los elementos determinados en los extractos de cada una de las tres fases del método BCR y la concentración en la fase residual sólida. Los recobrados en los extractos para el MRC (Tabla 5) oscilan entre 83 y 109\%, por lo que se puede considerar que existe una buena trazabilidad con respecto a los valores certificados. El V no se encuentra reportado.

\section{Vanadio}

La fracción mayoritaria de V (75-96\%) se asocia a la fase residual (Tabla 4), lo cual coincide con la baja extracción de este elemento por los métodos de digestión parcial. Estos resultados sugieren que el $\mathrm{V}$ pudiera ser de origen litogénico o estar vinculado al petróleo y carbón. ${ }^{38}$

\section{Níquel}

El Ni en las estaciones 3 y 6 se asocia fundamentalmente a la fase residual con 64 y 96\%, respectivamente. En la estación 3 alrededor 
Tabla 4. Concentración promedio \pm desviación estándar $\left(\mathrm{mg} \mathrm{kg}^{-1}\right.$ materia seca, $\left.\mathrm{n}=3\right)$ en las fracciones del método BCR y en la fracción residual de los sedimentos de la Bahía de Matanzas

\begin{tabular}{|c|c|c|c|c|c|c|}
\hline \multirow{2}{*}{ Estación } & \multirow{2}{*}{ Metal } & \multirow{2}{*}{$\mathrm{C}_{\mathrm{T}}^{\mathrm{a}}$} & \multicolumn{4}{|c|}{ Fracción (mg kg $\left.{ }^{-1}\right)$} \\
\hline & & & Intercambiable & Reducible & Oxidable & Residual \\
\hline \multirow{4}{*}{3} & $\mathrm{~V}$ & 67,7 & $1,7 \pm 0,1(2,5)$ & $7,2 \pm 0,9(10,6)$ & $7,8 \pm 0,5(11,5)$ & $\mathbf{5 1 , 0} \pm \mathbf{1 , 0}(75,3)$ \\
\hline & $\mathrm{Ni}$ & 22,6 & $2,5 \pm 0,3(11,1)$ & $1,2 \pm 0,2(5,3)$ & $4,3 \pm 0,5(19,0)$ & $14,6 \pm 1,0(64,6)$ \\
\hline & $\mathrm{Cu}$ & 27,9 & $0,30 \pm 0,05(1,1)$ & $0,05 \pm 0,01(0,2)$ & $2,6 \pm 1,0(9,3)$ & $\mathbf{2 5 , 0} \pm \mathbf{0 , 5}(89,6)$ \\
\hline & $\mathrm{Zn}$ & 55,5 & $3,8 \pm 0,5(6,8)$ & $13,1 \pm 0,5(23,6)$ & $24.4 \pm 0,9(44,0)$ & $14,1 \pm 1,1(25,6)$ \\
\hline \multirow{4}{*}{5} & $\mathrm{~V}$ & 84,1 & $0,20 \pm 0,05(0,2)$ & $2,3 \pm 0,7(2,7)$ & $3,1 \pm 0,5(3,7)$ & $\mathbf{7 8 , 5} \pm \mathbf{1 , 1}(93,3)$ \\
\hline & $\mathrm{Ni}$ & 98,3 & $2,1 \pm 0,2(1,6)$ & $1,2 \pm 0,1(1,2)$ & $\mathbf{5 3 , 3} \pm \mathbf{0 , 4}(54,2)$ & $\mathbf{4 1 , 8} \pm \mathbf{0 , 5}(42,5)$ \\
\hline & $\mathrm{Cu}$ & 18,1 & $0,30 \pm 0,01(1,6)$ & n.d $\mathrm{d}^{1}$ & $\mathbf{8 , 9} \pm \mathbf{1 , 0}(49,1)$ & $\mathbf{8 , 9} \pm \mathbf{0 , 5}(49,1)$ \\
\hline & $\mathrm{Zn}$ & 36,4 & $0,70 \pm 0,05(1,9)$ & $4,3 \pm 0,5(11,8)$ & $\mathbf{1 8 , 7} \pm \mathbf{0 , 5}(51,4)$ & $12,7 \pm 0,5(34,9)$ \\
\hline \multirow{4}{*}{6} & $\mathrm{~V}$ & 85,4 & $0,30 \pm 0,05(0,4)$ & $1,7 \pm 0,5(2,0)$ & $1,3 \pm 0,3(1,5)$ & $\mathbf{8 2 , 1} \pm \mathbf{1 , 0}(96,1)$ \\
\hline & $\mathrm{Ni}$ & 99,2 & $1,1 \pm 0,3(1,1)$ & $0,50 \pm 0,03(0,5)$ & $1,8 \pm 0,5(1,8)$ & $\mathbf{9 5 , 8} \pm \mathbf{1 , 1}(96,6)$ \\
\hline & $\mathrm{Cu}$ & 21,6 & $0,60 \pm 0,05(2,8)$ & n.d $d^{1}$ & $3,2 \pm 0,3(14,8)$ & $\mathbf{1 7 , 8} \pm \mathbf{1 , 1}(82,4)$ \\
\hline & $\mathrm{Zn}$ & 40,4 & $0,60 \pm 0,04(1,5)$ & $3,4 \pm 0,3(8,4)$ & $4,6 \pm 0,6(11,4)$ & $\mathbf{3 1 , 8} \pm \mathbf{1 , 0}(78,7)$ \\
\hline
\end{tabular}

${ }^{\mathrm{a}} \mathrm{C}_{\mathrm{T}}$ : Concentración total; ${ }^{1}$ No detectado; ( ): \% de recobrado.

Tabla 5. Concentración promedio \pm desviación estándar $\left(\mathrm{mg} \mathrm{kg}^{-1}\right.$ materia seca, $n=3$ ) en el material certificado de referencia BCR-701

\begin{tabular}{ccccc}
\hline \multirow{2}{*}{ Elemento } & Concentración & \multicolumn{3}{c}{ Fracción } \\
\cline { 3 - 5 } & & Intercambiable & Reducible & Oxidable \\
\hline $\mathrm{V}$ & Experimental & $0,58 \pm 0,0$ & $6,9 \pm 0,9$ & $5,1 \pm 0,2$ \\
& Certificada & - & - & - \\
$\mathrm{Ni}$ & Experimental & $15,9 \pm 0,2$ & $26,3 \pm 2,1$ & $13,1 \pm 1,3$ \\
& Certificada & $15,4 \pm 0,9$ & $26,6 \pm 1,3$ & $15,3 \pm 0,9$ \\
$\mathrm{Cu}$ & Experimental & $51,9 \pm 1,3$ & $135 \pm 10$ & $46,9 \pm 2,5$ \\
& Certificada & $49,3 \pm 1,7$ & $124 \pm 3$ & $55,2 \pm 4,0$ \\
$\mathrm{Zn}$ & Experimental & $192 \pm 7$ & $95,1 \pm 6,1$ & $50,6 \pm 1,5$ \\
& Certificada & $205 \pm 6$ & $114 \pm 5$ & $45,7 \pm 4,0$ \\
\hline
\end{tabular}

del $16 \%$ de Ni se encuentra repartido entre las fracciones intercambiable y reducible, y $19 \%$ en la oxidable. El porcentaje de Ni en las fracciones intercambiable-reducible-oxidable es de alrededor del $4 \%$ en la estación 6 , por lo que su efecto sobre la biota pudiera ser considerado prácticamente despreciable, a pesar de ser uno de los sedimentos más contaminados en Ni. En la estación 5, una fracción importante $(\sim 54,2 \%)$ se relaciona con la materia orgánica y los sulfuros, mientras que el $42,4 \%$ se asocia a la fracción residual. El contenido de $\mathrm{Ni}$ asociado a iones intercambiables y carbonatos es bajo (inferior al 3\%). Por tanto, la mayor cantidad de Ni en la estación 5 se encuentra asociado a las dos fases que son menos móviles y, a pesar del relativo alto contenido de Ni (supera el nivel del PEL) y lo que indican las guías consultadas, es muy poco probable que su efecto sea significativo sobre la biota. Un razonamiento similar es aplicable a la estación 6. El efecto real en cuanto a su posible removilización en ambas estaciones 5 y 6 es poco probable ya que se encuentra asociado mayoritariamente a fracciones poco móviles, fundamentalmente oxidable y residual.

Al comparar la extracción secuencial (Tabla 4) con los métodos de digestión parcial ensayados se observa que el método ASTM extrae la mayor parte de $\mathrm{Ni}$, entre 80 y $90 \%$ en las estaciones 3 y 5 . Esto indica que disuelve prácticamente el $\mathrm{Ni}$ presente en las cuatro fracciones del sedimento. La EE del método de ácido acético es inferior al 50\% en ambas estaciones, pero algo mayor que la EE considerando las dos primeras etapas del BCR (16,4 y 2,8\% para las estaciones 3 y 5 , respectivamente). Este resultado puede ser explicado por la mayor concentración del ácido en el método simple (25\% v/v) que remueve de manera efectiva la fracción adsorbida en las partículas de sedimento, así como la asociada a los carbonatos, oxhidróxidos de Fe y Mn y los enlazados a la materia orgánica y en muy poca extensión la fracción residual.

\section{Cobre}

Las fracciones más significativas de $\mathrm{Cu}$, según se aprecia en la Tabla 4, se asocian fundamentalmente a la fase residual $(49,1-89,6 \%)$ y en menor proporción a la materia orgánica y los sulfuros $(9,3$ y 49,1\%). Los niveles de $\mathrm{Cu}$ en la fracción oxidable se justifican por la elevada afinidad que presenta con la materia orgánica. ${ }^{39,40}$ Las concentraciones de $\mathrm{Cu}$ asociadas a óxidos de Fe-Mn y a iones intercambiables y carbonatos son prácticamente insignificantes $(<2,8 \%)$ en las tres estaciones estudiadas. Se puede concluir que el $\mathrm{Cu}$ en las estaciones 3, 5 y 6 tiene, al igual que el $\mathrm{Ni}$, una baja movilidad, debido a que se encuentra asociado fundamentalmente a la materia orgánica, los sulfuros y al retículo cristalino.

Por otra parte, el método ASTM disuelve gran parte del $\mathrm{Cu}$ presente en las estaciones 3 y 5 , por lo que, teniendo en cuenta que el recobrado obtenido fue muy cercano al $80 \%$ en estas estaciones, este método remueve todo el $\mathrm{Cu}$ presente en las tres primeras fracciones del BCR y una parte considerable del que se encuentra en la fracción residual.

En las estaciones 3 y 5, el método del ácido acético disuelve sólo la fracción de Ni, $\mathrm{Cu}$ y Zn adsorbida en las partículas del sedimento, así como la asociada a la fase intercambiable. Para la estación 6 se lixivió por el método ASTM aproximadamente $30 \%$ del $\mathrm{Cu}$ total y alrededor del $20 \%$ por el método del ácido acético. Según los resultados del BCR para la estación 6, el método ASTM digiere totalmente las fracciones intercambiable, reducible y oxidable (en las cuales la proporción de $\mathrm{Cu}$ es baja $\approx 17 \%$ ), y actúa además en cierta medida sobre la fase residual. La acción del método del ácido acético es similar a la del ASTM, sólo que su extensión a la fase residual es ligeramente inferior.

\section{Zinc}

Las mayores concentraciones (Tabla 4) se encuentran en la fase oxidable y residual para las tres estaciones estudiadas. En la estación 
3 un $44 \%$ del Zn total se enlaza a la fase oxidable, mientras 23,6\% se asocia a los oxhidróxidos de Fe y Mn, y 6,8\% se encuentra adsorbido en las partículas de sedimentos o asociado a carbonatos. Para la estación 5, el 51\% del Zn se encuentra en la fracción oxidable y alrededor del $35 \%$ permanece unido al retículo cristalino. En la estación 6, la mayor parte del $\mathrm{Zn}$ presente $(78,7 \%)$ se encuentra asociado a la fase residual, mientras que alrededor del $20 \%$ se distribuye entre las fracciones reducible y oxidable. De acuerdo a los elevados porcentajes de $\mathrm{Zn}$ en la fracción residual, para las tres estaciones estudiadas, se puede concluir que, una parte significativa del metal en los sedimentos es de origen litogénico, sin embargo se debe destacar el incremento de las formas móviles (intercambiable y reducible) en estos sitios donde el contenido de $\mathrm{Zn}$ en las fracciones de mayor movilidad se encuentra aproximadamente entre el 10 y el $30 \%$ del $\mathrm{Zn}$ total, lo que evidencia la influencia antrópica. Estos resultados demuestran que la movilidad del $\mathrm{Zn}$ puede modificarse debido a cambios en las condiciones oxidantes del medio. ${ }^{41}$

Comparando los resultados de las extracciones de una sola etapa con los resultados del BCR se observa que para las estaciones 3 y 5 el ácido acético disuelve totalmente los metales adsorbidos en las partículas de sedimento, así como los que se encuentran asociados a los carbonatos y oxhidróxidos de Fe y Mn y, actúa poco sobre los enlazados a la materia orgánica ya que se extrajo entre 10 y $50 \%$. El método ASTM, por otra parte, disuelve totalmente las tres primeras fracciones y una porción considerable de la fase residual, pues las extracciones son superiores al 90\%. Para la estación 6 la acción de ambos métodos de digestión es similar, disolviendo prácticamente las fracciones intercambiable, reducible y oxidable de forma total.

En general, la proporción de metales disueltos en cada fase es variable y depende tanto de la muestra, como del elemento analizado. Para los sedimentos estudiados el mayor porcentaje de extracción de $\mathrm{Ni}, \mathrm{Cu}, \mathrm{Zn}$ y V se logra en la fase 3 del proceso de extracción secuencial. Esto permite sugerir que los metales estudiados deben estar fundamentalmente asociados a la materia orgánica y al material sulfhídrico, lo que corrobora el papel dominante de la materia orgánica en la distribución y dispersión de los metales pesados en el medio ambiente debido a su elevada capacidad para complejar cationes. ${ }^{42}$

Los resultados del BCR permitieron diferenciar las muestras 3 y 5 respecto a la distribución de los metales en las diferentes fases del sedimento. Esta diferenciación no pudo ser reconocida por el método ASTM que extrajo, prácticamente, todo el contenido de $\mathrm{Cu}$, $\mathrm{Ni}$ y Zn de ambas muestras. El BCR demuestra que en la muestra 3 el contenido principal de $\mathrm{Ni}$ y $\mathrm{Cu}$ se encuentra en la fase residual, mientras que el $\mathrm{Zn}$ se distribuye en las fracciones oxidable y residual, con cierto incremento en la oxidable. Sin embargo, en la muestra 5 el $\mathrm{Cu}$, Ni y $\mathrm{Zn}$ se asocian principalmente y en cantidades similares a las fases oxidable y residual.

En la muestra 6 el contenido principal de todos los elementos se asocia a la fase residual. Esta mayor cantidad de elementos asociados a la fase residual no puede ser extraída totalmente por el método ASTM, como se demostró previamente, lo que indica que una parte de ese contenido en la fase residual está muy fuertemente ligado al retículo cristalino. Esto se confirma con el hecho de que el contenido extraído por el ASTM y el método acido acético es similar en esta muestra. Es decir, se asocian los elementos en esta muestra a fracciones bien diferenciadas: la residual que no se extrae por los métodos parciales empleados y otra, que comprende la suma de varias fases y que es igualmente extraíble por los métodos de digestión parciales ensayados.

\section{CONCLUSIONES}

Las estaciones con mayor contaminación, fundamentalmente por Ni y V, son 5 y 6 , seguidas por la 3 . La extracción secuencial en sedimentos de las tres zonas más contaminadas demostró que la mayor parte de $\mathrm{Ni}, \mathrm{Cu}, \mathrm{Zn}$ y V se encuentra en las fracciones más estables del sedimento: la residual (origen litogénico) y la oxidable, es decir, con una notable baja movilidad y disponibilidad para el medio. De los metales estudiados, el Zn es el elemento químico más susceptible a incrementar su potencial de removilización y pasar hacia la columna de agua al ocurrir cambios en los parámetros físico-químicos del sistema.

\section{MATERIAL SUPLEMENTARIO}

Disponible en http://quimicanova.sbq.org.br, en archivo pdf, con acceso libre. Localización geográfica de los sitios de muestreo de la bahía de Matanzas en la Figura 1S.

\section{AGRADECIMIENTOS}

A la Asociación Universitaria Iberoamericana de Postgrado (AUIP) y al Aula Universitaria Iberoamericana de la Universidad de Cádiz, España, el financiamiento asignado para la realización de esta investigación.

\section{REFERENCIAS}

1. González, H.; Brugmann, L.; Chemistry and Ecology 1989, 4, 37.

2. Tur, A.; Martin, A.; Palacios, F.; Beltrán, J.; Ramírez, M.; Regadera, R.; Ruíz, F.; Informe Final Proyecto: Evaluación y Control de la Contaminación Marina en las Bahías de Cienfuegos, Zona Cárdenas-Varadero, Matanzas, Mariel, Nuevitas, Nipe, Puerto Padre y Santiago de Cuba, 2000.

3. Ruíz, F.; Pérez, M.; Beltràn, J.; Mancebo, H.; Regadera, R.; Martín, A.; Ramírez, M.; Solar, F.; Rev. Invest. Mar. 2008, 29, 13.

4. Šcancar, J.; Milacic, R.; Strazar, M.; Burica, O.; Sci. Total Environ. 2000, 250, 9 .

5. Cuong, D. T.; Obbard, J. P.; Appl. Geochem. 2006, 21, 1335.

6. Rao, C. R. M.; Sahuquillo, A.; Lopez Sanchez, J. F.; Water, Air, Soil Pollut. 2008, 189, 291.

7. DIN 38414-S4, German standard methods for the examination of water, waste wáter and sludge; Group $S$ (sludge and sediments). Determination of leachability by water (S4), 1984.

8. EPA; Method 3050B, Acid digestion of sediments, sludges and soils, 1996.

9. EPA; Method 3051A, Microwave-assisted acid digestion of sediments, sludges, soils, and oils, 2007.

10. ASTM; D3974, Standard practices for extraction of trace elements from sediments, 2003.

11. ISO 11466; Soil quality: Extraction of trace elements soluble in aqua regia, 1995.

12. Bettiol, C.; Stievano, L.; Bertelle, M.; Delfino, E. A.; Appl. Geochem. 2008, 23, 1140.

13. Burton, E.; Phillips, I.; Hawler, D.; J. Environ. Qual. 2005, 34, 263.

14. Moreda Piñeiro, J.; Alonso Rodríguez, E.; Lopez Mahía, P.; Muniategui Lorenzo, S.; Prada Rodríguez, D.; Moreda Piñeiro, A.; Bermejo Barrera, A.; Bermejo Barrera, P.; Spectrochim. Acta, Part B 2006, 61, 1304.

15. Sahuquillo, A.; Rigor, A.; Rauret, G.; J. Environ. Monit. 2002, 4, 1003.

16. Filippi, M.; Dousova, B.; Machovic, V.; Czech. Republic. Geoderma 2007, 139, 154

17. Icart, M. P.; Villanueva, M.; Toledo, C.; Rodríguez, J.; Behar, M.; Pomares, M.; Mar. Environ. Res. 2011, 72, 60.

18. Tessier, A.; Campbell, P. G. C.; Bissom, M.; Anal. Chem. 1979, 51, 844.

19. Sahuquillo, A.; López-Sánchez, J. F.; Rubio, R.; Rauret, G.; Thomas, E. A.; Davidson, C. M.; Ure, A. M.; Anal. Chim. Acta 1999, 382, 317. 
20. Pérez, G.; Tesis de Doctorado, Universidad Autónoma de Barcelona España, 2005.

21. Cuong, D. T.; Obbard, J. P.; Appl. Geochem. 2006, 21, 1335.

22. Svete, P.; Mila cǐc c, R.; Pihlar, B.; J. Environ. Monit.2000, 3, 586.

23. Casado-Martínez, M. C.; Buceta, J. L.; Belzunce, M. J.; DelValls, T. A.; Environ. Int. 2006, 32, 388.

24. Rodríguez, M. R.; Tesis de Doctorado, Universidad de Cádiz, España, 2003.

25. Tomllinson, D. C.; Wilson, J. G.; Harris, C. R.; Jeffrey, D. W.; Helgoländer Wissenschaftliche Meeresuntersuchungen 1980, 33, 566.

26. Gracia,I.; Morillo, J.; Usero, J.; Actas del Congreso Chemical Industry and Environment IV, Gran Canaria, España, 2003.

27. Usero, J.; Gracia, A.; Leal, A.; Fraidías, J.; Calidad de las aguas y sedimentos del Litoral Andaluz, Editorial Junta de Andalucía: Sevilla, 2000

28. Long, E. R.; MacDonald, D. D.; Smith, S. L.; Calder, F. D.; Env. Manag. 1995, 19, 81

29. Förstner, U.; Wittman, G.; Metal pollution in the aquatic environment, Springer Verlag: Berlin, 1979.

30. García, J.; Acuña, J.; Vargas, J.; Rev. Biol. Trop. 2004, 52, 51.

31. ISO 5667/19; Calidad del agua. Muestreo. Parte 19: Guía para el muestreo de sedimentos marinos, 2004.
32. ISO 5667/15; Calidad del agua. Muestreo. Parte 15: Guía sobre conservación y manipulación de muestras de lodo y sedimento, 1999.

33. UNEP/IOC/IAEA; Reference Methods for Marine pollutions Studies, 1995, No. 63.

34. Loring, D. H.; Rantala, R. T. T.; Earth-Science Reviews 1992, 32, 235.

35. Ajlec, R.; Cop, M.; Stupar, J.; Analyst 1988, 113, 585.

36. Rauret, G.; López-Sánchez, J. F.; Sahuquillo, A.; Rubio, R.; Davidson, C. M.; Ure, A. M.; Quevauviller, P.; J. Environ. Monit. 1999, 1, 57.

37. SQAGs - Sediment Quality Assessment Guidelines 2000, chap. 6.

38. Amorim, F. A. C.; Welz, B.; Costa, A. C. S.; Lepri, F. G.; Goreti, M.; Vale, R.; Ferreira, S. L. C.; Talanta 2007, 72, 349.

39. Azevedo, M. L.; Ferraceiu, L. R.; Guimaraes, L. R.; Sci. Agric. 2003, 60,793 .

40. Zhou, L. X.; Wong, J. W. C.; J. Environ. Qual. 2001, 30, 878.

41. Nemati, K.; Kartini, N.; Sobhanzader, E.; Radzi, M.; Microchem. J. 2009, 92, 165

42. Douglas, G. B.; Hart, B. T.; Beckett, R.; Gray, C. M.; Oliver, R. L.; Aquat. Geochem. 1999, 5, 167. 